\title{
SINGLE SPIKE OPERATION IN SPARC SASE-FEL
}

\author{
I. Boscolo, F.Castelli, S. Cialdi, V. Petrillo, Università di Milano, Milano (Italy) \\ A.Bacci, L.Serafini, INFN, Milano (Italy) \\ R. Bonifacio, M. Boscolo, M. Ferrario, C. Vaccarezza, LNF, INFN, Frascati (Italy) \\ L. Giannessi,C. Ronsivalle, ENEA Frascati, (Italy) \\ L. Palumbo, M. Serluca, Università La Sapienza Roma (Italy) \\ J. Rosenzweig. S. Reiche, UCLA, LA (USA)LNF, INFN, Frascati (Italy)
}

\section{Abstract}

The single spike operation regime has been analysed in the case of the SPARC injector and free-electron-laser. Four different beams at $50 \mathrm{pC}$ are studied, with different production condition and performance.

\section{INTRODUCTION}

In the FEL emission two different regimes occur depending on the length $\mathrm{L}_{b}$ of the beam.

If the $\mathrm{L}_{\mathrm{b}}$ is larger than $2 \pi$ times the cooperation length $\mathrm{L}_{\mathrm{c}}$, the radiation presents a longitudinal structure constituted by several chaotic peaks, while, if the length of the beam is shorter than $2 \pi \mathrm{L}_{\mathrm{c}}$, the emission produces a radiation pulse shaped in one single spike [1]. This regime occurs because the radiation emitted by the electrons, travelling from the tail towards the head of the beam, covers all the distance inside the bunch in a time shorter than few gain times, correlating all the particles. The properties of this regime are well-known in $1 \mathrm{~d}$ : however, the study of single-spike ultra-short radiation in the $X$ rays range [2], as well as in the visible light [3], by means of start-to-end simulations from the photocathode to the end of the undulator, has shown that transverse and non-homogeneity effects due to radiation diffraction and to non-ideal characteristics of the electron beam such as emittance and energy spread change considerably the properties of the emission process. A fundamental problem is also how to produce a suitable beam. In the second part of this paper, the analysis of the $3 \mathrm{~d}$ scaling law is presented. Then we present some numerical startto-end FEL simulations made in the case of the SPARC FEL, for some different beam regimes at $50 \mathrm{pC}$. The performance of the various bunches are compared and the most interesting of them are discussed.

\section{SCALING LAW}

The single spike operation requires that the beam length $\mathrm{L}_{\mathrm{b}}$ satisfies the following requirement:

$$
\mathrm{L}_{\mathrm{b}} \leq 2 \pi \mathrm{L}_{\mathrm{c}}
$$

with $\mathrm{L}_{\mathrm{c}}=\mathrm{L}_{\mathrm{c} 1 \mathrm{~d}}(1+\eta)$

where: $L_{c l d}=\lambda /(\sqrt{3} 4 \pi \rho)$ and $\eta$ is defined as in [4] :

$$
\begin{aligned}
\eta= & 0.45 \eta_{\mathrm{d}}{ }^{0.57}+0.55 \eta_{\varepsilon}{ }^{1.6}+3 \eta_{\gamma}{ }^{2}+0.35 \eta_{\varepsilon}{ }_{\varepsilon}^{2.9} \eta_{\gamma}{ }^{2.4} \\
& +51 \eta_{\mathrm{d}}{ }^{0.95} \eta_{\gamma}{ }^{3}+5.4 \eta_{\mathrm{d}}{ }^{0.7} \eta_{\varepsilon}{ }^{1.9}+1140 \eta_{\mathrm{d}}{ }^{2.2} \eta_{\varepsilon}{ }^{2.9} \eta_{\gamma}{ }^{3.2},
\end{aligned}
$$

with $\eta_{\mathrm{d}}=\mathrm{L}_{\mathrm{g} 1 \mathrm{~d}} \lambda /\left(4 \pi \sigma_{\mathrm{x}}^{2}\right)$ term that accounts for radiation diffraction, $\eta_{\varepsilon}=\frac{4 \pi \mathrm{L}_{\mathrm{gld}} \varepsilon_{\mathrm{n}, \mathrm{x}}^{2}}{\sigma_{\mathrm{x}}^{2} \gamma^{2} \lambda}$ for the emittance and $\eta_{\gamma}=4 \pi \frac{\mathrm{L}_{\mathrm{gld}}}{\lambda_{\mathrm{u}}} \frac{\delta \gamma}{\gamma}$ for the energy spread effects. In these last expressions $\mathrm{L}_{\mathrm{g} 1 \mathrm{~d}}=\lambda_{\mathrm{v}} /(\sqrt{3} 4 \pi \rho)$ is the $1 \mathrm{~d}$ gain length, $\varepsilon_{\mathrm{n}, \mathrm{x}}$ the normalized transverse emittance, $\delta \gamma / \gamma$ the energy spread and $\lambda$ is the radiation wavelength given by the resonance condition $\lambda=\frac{\lambda_{\mathrm{u}}\left(1+\mathrm{a}_{\mathrm{w}}^{2}\right)}{2 \gamma^{2}}$.

The FEL parameter $\rho$, in terms of the beam average current I, of the radial r.m.s dimension $\sigma_{\mathrm{x}}$ of the beam, of the undulator parameter $\mathrm{K}_{0}=\sqrt{2} \mathrm{a}_{\mathrm{w}}$ and period number $\mathrm{k}_{\mathrm{u}}=2 \pi / \lambda_{\mathrm{u}}$, of the Lorentz factor of the beam $\gamma$ can be written as:

$$
\rho=\left[\frac{1}{16} \frac{\mathrm{I}}{\mathrm{I}_{\mathrm{A}}} \frac{\mathrm{K}_{\mathrm{o}}^{2}[\mathrm{JJ}]^{2}}{\gamma^{3} \sigma_{\mathrm{x}}^{2} \mathrm{k}_{\mathrm{u}}^{2}}\right]^{1 / 3}
$$

where $\mathrm{I}_{\mathrm{A}}=17 \mathrm{KA}$ is the Alfven current and $\mathrm{JJ}=\left(\mathrm{J}_{0}(\xi)\right.$ $\left.\mathrm{J}_{1}(\xi)\right)$, J's are Bessel function of argument $\xi=\frac{\mathrm{a}_{\mathrm{w}}^{2}}{2\left(1+\mathrm{a}_{\mathrm{w}}^{2}\right)}$.

In (4) the current $I$ is defined as $I=c Q / L_{b}$ with $L_{b}$ the whole beam length if the beam current is flat top, or $\mathrm{L}_{\mathrm{b}}=\sqrt{2 \pi} \sigma_{\mathrm{z}}$ with $\sigma_{\mathrm{z}}$ the FWHM length, if the longitudinal beam profile is Gaussian.

The single spike condition is:

$$
\mathrm{L}_{\mathrm{b}}=2 \pi \mathrm{L}_{\mathrm{c} 1 \mathrm{~d}}(1+\eta)
$$

and the $\mathrm{Q}$ vs $\mathrm{L}_{\mathrm{b}}$ scaling law becomes

$$
\mathrm{Q}=\left(\frac{\pi^{2} \mathrm{I}_{\mathrm{A}}}{3 \sqrt{3} \mathrm{c}}\right)\left(\frac{\lambda_{\mathrm{u}}\left(1+\mathrm{a}_{\mathrm{w}}^{2}\right)^{3}}{\mathrm{~K}_{0}{ }^{2}[\mathrm{JJ}]^{2}}\right)\left(\frac{\sigma_{\mathrm{x}}^{2}}{\mathrm{~L}_{\mathrm{b}}^{2} \gamma^{3}}\right)(1+\eta)^{3}
$$

where in the factor $\eta$ a further irrational dependence on $Q$ and $\mathrm{L}_{\mathrm{b}}$ is contained.

The solution of (5) for four different beams obtained with four different beam lines at $50 \mathrm{pC}$ is presented in Fig 1. The beams present different values of emittance, energy spread, current profile, longitudinal width and transverse 
dimension. Their position in the $\mathrm{Q}$ vs $\sigma_{\mathrm{z}}$ plane is represented by green stars in Fig 1, together with single spike operation curves at different $\sigma_{\mathrm{x}}$.

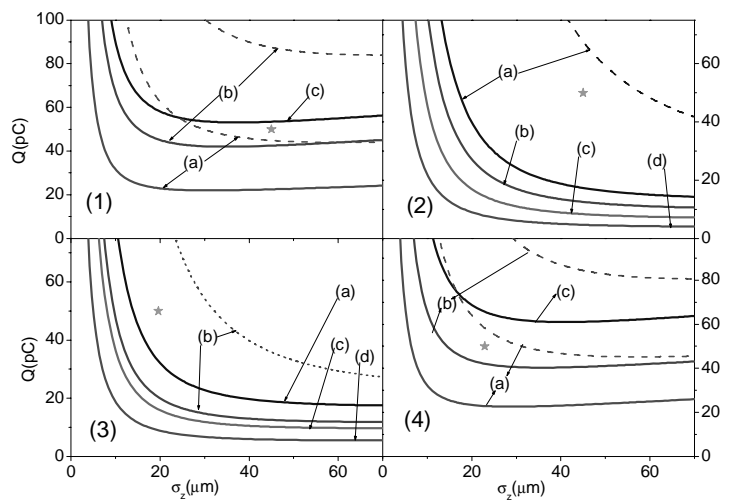

Figure 1:single spike scaling law: solid: 1 spike, dashed: 2 spikes for: (1) beam 1 (a) $\sigma_{x}=50 \mu \mathrm{m}$, (b) $\sigma_{x}=100 \mu \mathrm{m}$ (c) $\sigma_{x}=126 \mu \mathrm{m}$. (2) beam 2 (a) $\sigma_{\mathrm{x}}=200 \mu \mathrm{m}$ (b) $\sigma_{\mathrm{x}}=150 \mu \mathrm{m}$ (c) $\sigma_{x}=104 \mu \mathrm{m}$, (d) $\sigma_{x}=50 \mu \mathrm{m}$. (3) beam 3: (a) $\sigma_{x}=150$ $\mu \mathrm{m}$,(b) $\sigma_{\mathrm{x}}=100 \mu \mathrm{m}$ (c) $\sigma_{\mathrm{x}}=80 \mu \mathrm{m}$ (d) $\sigma_{\mathrm{x}}=50 \mu \mathrm{m}$. (4): beam 4 (a) $\sigma_{x}=150 \mu \mathrm{m}$, (b) $\sigma_{x}=100 \mu \mathrm{m}$, (c) $\sigma_{x}=50 \mu \mathrm{m}$.

Table 1

\begin{tabular}{|l|l|l|l|l|l|}
\hline beam & $\phi\left(^{\circ}\right)$ & $\begin{array}{l}\varepsilon_{\mathrm{n}} \\
\mu \mathrm{m}\end{array}$ & $\begin{array}{l}\Delta \mathrm{E} / \mathrm{E} \\
\%\end{array}$ & $\begin{array}{l}\sigma_{\mathrm{z}} \\
\mu \mathrm{m}\end{array}$ & $\begin{array}{l}\mathrm{I}_{\text {peak }} \\
\text { A }\end{array}$ \\
\hline 1 & -84.5 & 0.47 & 2.35 & 45 & 120 \\
\hline 2 & -95 & 0.45 & 0.69 & 45 & 120 \\
\hline 3 & -89.9 & 0.63 & 0.97 & 20 & 300 \\
\hline 4 & -91.4 & 2. & 2.1 & 22.8 & 430 \\
\hline
\end{tabular}

\section{OPERATION AT 50 pC}

Four different beams generated and driven in the SPARC line [5] have been analysed, characterized substantially by different values of the injection angle $\phi$ in the first accelerating structure. The RF compression method has been used. The phase spaces together with the current profile are presented in Fig 2. The first beam (fig. 2, window (1)) has been obtained in the standard SPARC operation regime, scaling the parameters from the $1 \mathrm{nC}$ working point by means of the scaling law at the cathode $\sigma_{\mathrm{xyz}} \sim \mathrm{Q}^{1 / 3}[6]$, and using therefore a laser pulse length of $\sigma_{\mathrm{t}}=1$ psec, illuminating a region of $\mathrm{R}=0.4 \mathrm{~mm}$. The injection angle $\phi$ was $-84.5^{\circ}$. The beam was compressed at $\sigma_{\mathrm{z}}=45 \mu \mathrm{m}$, with a peak current of 120 A.The simulations of the beams 1,2 and 4 was done with Parmela [7], while case 3 was simulated by ASTRA [8]. was obtained in the blow out regime with a laser pulse of $0.2 \mathrm{psec}$ and an injection angle of $-95^{\circ}$ in the overcompression condition. The final beam length is again 45 $\mu \mathrm{m}$ and the peak current $120 \mathrm{~A}$, but the energy spread and the transverse dimension are smaller and the current has a different shape.

The last two beams (3, shown in Fig. 2 , window (3) and 4, Fig. 2, window (4)) have been injected around the 02 Synchrotron Light Sources and FELs maximum compression phase (respectively $-89.9^{\circ}$ and $91.4^{\circ}$ ), obtaining more peaked currents. The difference between them is that beam (3) has been optimized with the genetic algorithm [9], leaving free the intensities of the 12 magnetic coils of the first structure and the injection angles in the last two structures (respectively $34.9^{\circ}$ and -2.8). These adjunctive degrees of freedom have permitted to obtain a current very much larger than the first two cases, only a bit smaller than case (4), but with a better control of emittance and of energy spread. The last beam 4 belongs to the high current operation regime, widely explained in Ref [3].

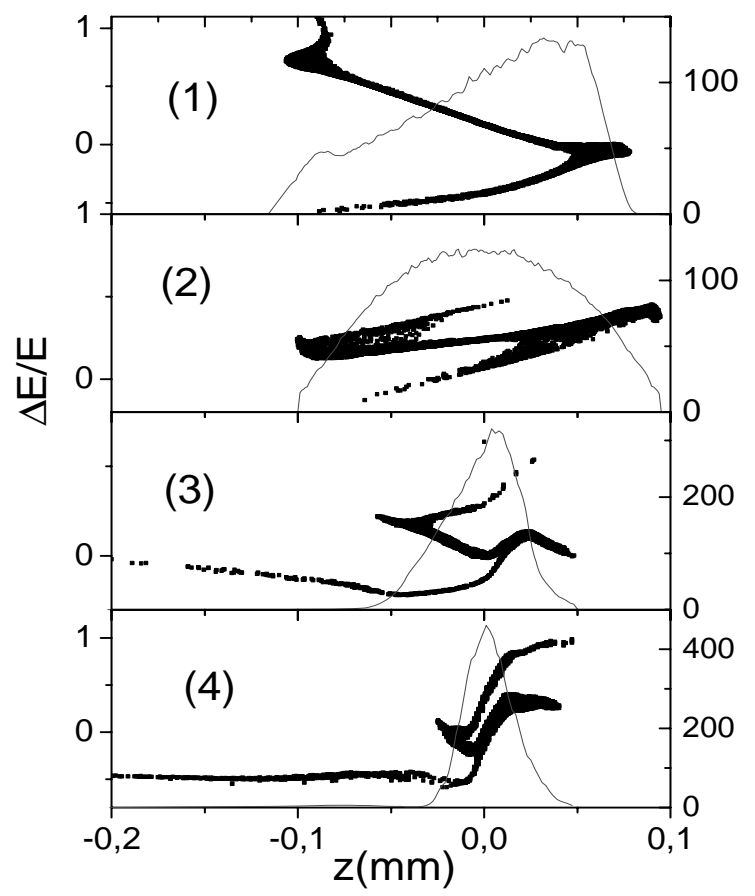

Figure 2: Left axis: Phase space $\Delta \mathrm{E} / \mathrm{E}$ in $\%$ vs $\mathrm{z}(\mathrm{mm})$. Right axis: current (a) vs $\mathrm{z}(\mathrm{mm})$ for (1): beam 1, (2) beam 2, (3) beam 3 and (4) beam 4 .

In table 1 the different characteristics of the beams are summarized. In the last two cases the effect of the large longitudinal space charge forces is that of prevent the complete overcompression, the particles being pushed forward by the electric repulsive forces applied by the slices behind. The result is a folding in the phase space in correspondence of the maximum density with inversion of the energy phase correlation.

\section{FEL SIMULATIONS}

The optimum focusing condition for the four beams has been deduced by the scaling law shown in Fig. 1. Values of $\sigma_{\mathrm{x}}$ and $\sigma_{\mathrm{x}}$ leading to the single spike operation with the maximum focalization (corresponding to the maximum energy extraction) have been used. For the beam (1) the condition of single spike occurs for $\sigma_{x}=100 \mu \mathrm{m}$. In fact in 
Fig 1 (1) the green star is positioned near curve (b) solid line ( 1 spike, and (b) $\left.\sigma_{x}=100 \mu \mathrm{m}\right)$ and well above curve (b) dashed line ( 2 spikes, $\left.\sigma_{\mathrm{x}}=100 \mu \mathrm{m}\right)$. As the beam at 12 meter has $\sigma_{\mathrm{x}}=126 \mu \mathrm{m}$, it has been transversally matched to the undulator entrance by means of the SPARC transfer line constituted by two triplets far $3.8 \mathrm{~m}$. The second beam has $\sigma_{\mathrm{x}}=104 \mu \mathrm{m}$, so it can be not focused. Beam 3 presents $\sigma_{\mathrm{x}}=79 \mu \mathrm{m}$ at $12 \mathrm{~m}$, so it has to be matched, as well as beam 4 that exits from the linac with $\sigma_{x}=208 \mu \mathrm{m}$.

The undulator and lattice characteristics are: 6 undulator section of $2.15 \mathrm{~m}, \lambda_{\mathrm{u}}=2.8 \mathrm{~cm}, \mathrm{a}_{\mathrm{w}}=1.51$, $\mathrm{dB} / \mathrm{dz}=8$. $\mathrm{T} / \mathrm{m}$. Radiation with wavelength of $500 \mathrm{~nm}$ is produced.

In table 2 the most significant radiation characteristics are presented for various values of $\sigma_{\mathrm{x}}$. The FEL simulations have been done with GENESIS 1.3 [10] .

Table 2: radiation properties: $\mathrm{N}_{\mathrm{sp}}$ : number of radiation spikes, E: total energy of the pulse, div: radiation divergence, $\sigma_{\text {rad }}^{z,}$ :radiation length, bw: normalized bandwidth

\begin{tabular}{|l|l|l|l|l|l|l|l|}
\hline beam & $\begin{array}{l}\boldsymbol{\sigma}_{\mathbf{x}} \\
\boldsymbol{\mu} \mathbf{m}\end{array}$ & $\mathbf{N}_{\mathbf{s p}}$ & $\begin{array}{l}\mathbf{P}_{\max } \\
\mathbf{G W}\end{array}$ & $\begin{array}{l}\mathbf{E} \\
\mu \mathbf{J}\end{array}$ & $\begin{array}{l}\mathbf{d i v} \\
\mathbf{m r a d}\end{array}$ & $\begin{array}{l}\boldsymbol{\sigma}_{\text {rad }}^{\mathbf{\mu}} \\
\boldsymbol{\mathbf { m }}\end{array}$ & $\begin{array}{l}\mathbf{b w} \\
\boldsymbol{\%}\end{array}$ \\
\hline 1 & 104 & $1-2$ & 0.13 & 23 & 1.7 & 30 & 1 \\
& 50 & $1-3$ & 0.095 & 30 & 1 & 70 & 1 \\
\hline 2 & 104 & $1-2$ & 0.037 & 10 & 0.7 & 50 & 0.8 \\
& 50 & 3 & 0.09 & 30 & 0.6 & 250 & 1 \\
\hline 3 & 104 & 1 & 0.16 & 16.7 & 1 & 20 & 1 \\
& 79 & $1-2$ & 0.32 & 44 & 1 & 40 & 1 \\
& 50 & 3 & 0.37 & 56 & 0.9 & 60 & 0.8 \\
\hline 4 & 125 & 1 & 0.41 & 39.6 & 1.2 & 15 & 1.1 \\
& 100 & $1-2$ & 0.7 & 50.8 & 2 & 20 & 1.2 \\
& 70 & $1-2$ & 0.8 & 68.4 & 1.6 & 25 & 1.3 \\
\hline
\end{tabular}

In figures 3 and 4 the radiation evolution is presented in the plane $\mathrm{z}(\mathrm{m})$ vs $\mathrm{s}(\mu \mathrm{m})$, together with the pulse shape $\mathrm{P}(\mathrm{W})$ vs $\mathrm{s}$ at 11 meter in the undulator. The cases presented are: beam 1, $\sigma_{x}=104 \mathrm{~mm}$, and beam $4, \sigma_{x}$ $=125 \mathrm{~mm}$. As shown, the predictions of the scaling law are respected. The analysis shows that there is large margin of choice in the single spike operation. The maximum compression regime leads to minima pulse lengths, large peak power and total energy. However, the operation in this regime requires a tight control of the beam line elements (as for instance the magnetic field intensity in the coils of the first structure) and of the injection angles for avoiding the formation of tails that degrade the beam quality and for controlling emittance and energy spread. These last quantities are not demanding for the single spike occurrence, but determine some of the pulse characteristics as, for instance, the divergence and the spectrum.
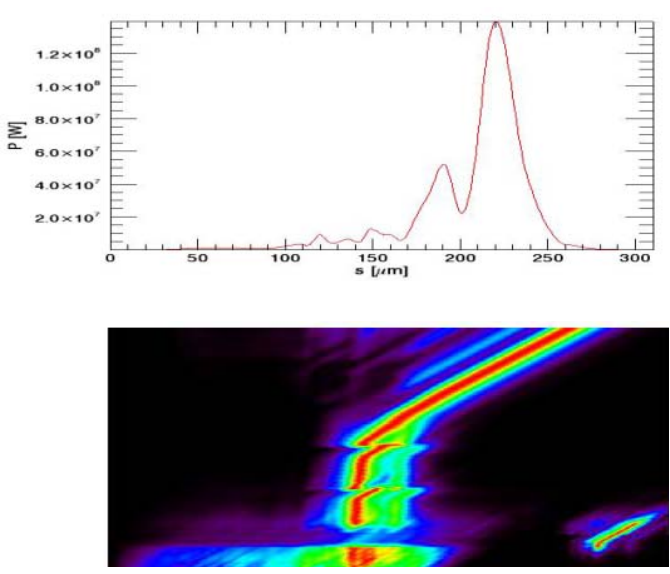

Figure 3: beam 1, $\sigma_{\mathrm{x}}=104 \mu \mathrm{m}$ : pulse shape $\mathrm{P}(\mathrm{W})$ vs $\mathrm{s}(\mu \mathrm{m})$ at $11 \mathrm{~m}$. Normalized level curves in the plane $\mathrm{z}(\mathrm{m})$ vs s $(\mu \mathrm{m})$.

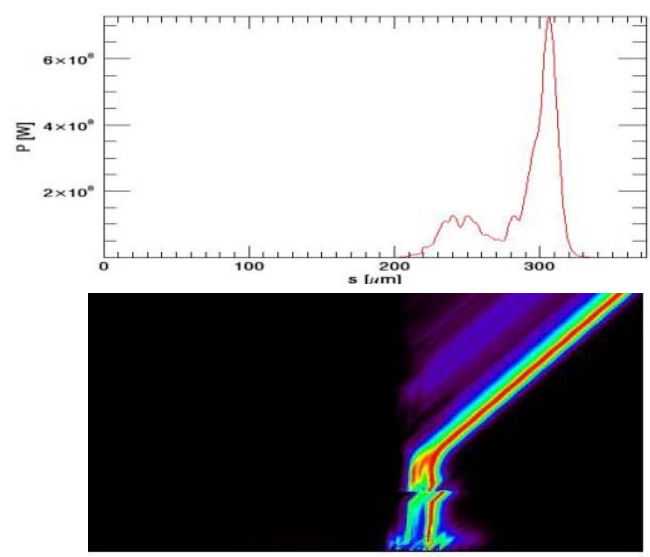

Figure 4: beam 4, $\sigma_{\mathrm{x}}=100 \mu \mathrm{m}$ : pulse shape $\mathrm{P}(\mathrm{W})$ vs $\mathrm{s}(\mu \mathrm{m})$ at 11 meterr. Normalized level curves in the plane $\mathrm{z}(\mathrm{m})$ vs s $(\mu \mathrm{m})$.

\section{REFERENCES}

[1] R. Bonifacio et al. PRL 73 (1994) 70

[2] J. Rosenzweig et al. NIMA (2008) in press

[3] M.Boscolo et al, NIMA (2008) doi:10.1016/j.nima.2008.04.074

[4] M. Xie in Proc. of the 1995 PAC, IEEE, Piscataway, NJ,1995, p.183

[5] M. Ferrario et al. PRL 99 (2007) 234801

[6] M. Boscolo et al.: SPARC BD-note BD-07-006

[7] L.M. Young, LANL Report No.LA-UR-96-1835

[8] K. Floetmann, ASTRA: http://desy.de/ mpyflo/Astra_dokumentation/

[9] A. Bacci et al., NIMB 263 (2007) 488

[10] S. Reiche: NIM A 429 (1999) 243 